\title{
Applications of Sensitivity Analysis to Uncertainty Quantification in Variably Saturated Flow
}

C.S. Woodward, K.E. Grant, R. Maxwell

This article was submitted to XIV International Conference on Computational Methods in Water Resources, Amsterdam, The Netherlands, June, 2002

Lawrence

January 1, 2002

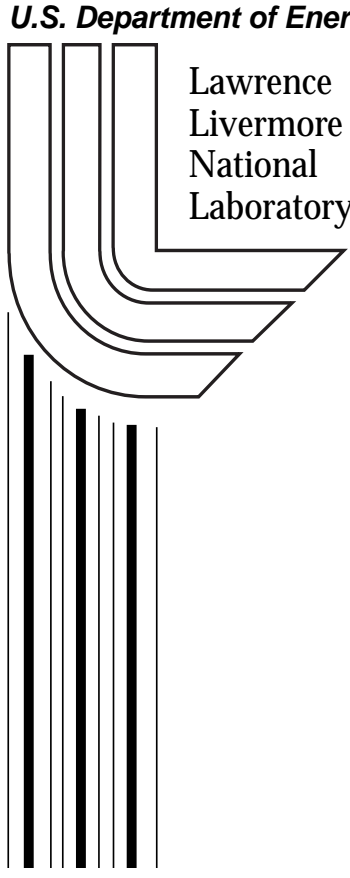




\section{DISCLAIMER}

This document was prepared as an account of work sponsored by an agency of the United States Government. Neither the United States Government nor the University of California nor any of their employees, makes any warranty, express or implied, or assumes any legal liability or responsibility for the accuracy, completeness, or usefulness of any information, apparatus, product, or process disclosed, or represents that its use would not infringe privately owned rights. Reference herein to any specific commercial product, process, or service by trade name, trademark, manufacturer, or otherwise, does not necessarily constitute or imply its endorsement, recommendation, or favoring by the United States Government or the University of California. The views and opinions of authors expressed herein do not necessarily state or reflect those of the United States Government or the University of California, and shall not be used for advertising or product endorsement purposes.

This is a preprint of a paper intended for publication in a journal or proceedings. Since changes may be made before publication, this preprint is made available with the understanding that it will not be cited or reproduced without the permission of the author.

This work was performed under the auspices of the United States Department of Energy by the University of California, Lawrence Livermore National Laboratory under contract No. W-7405-Eng-48.

This report has been reproduced directly from the best available copy.

Available electronically at http://www.doc.gov/bridge

Available for a processing fee to U.S. Department of Energy

And its contractors in paper from

U.S. Department of Energy

Office of Scientific and Technical Information

P.O. Box 62

Oak Ridge, TN 37831-0062

Telephone: (865) 576-8401

Facsimile: (865) 576-5728

E-mail: reports@adonis.osti.gov

Available for the sale to the public from

U.S. Department of Commerce

National Technical Information Service

5285 Port Royal Road

Springfield, VA 22161

Telephone: (800) 553-6847

Facsimile: (703) 605-6900

E-mail: orders@ntis.fedworld.gov

Online ordering: http://www.ntis.gov/ordering.htm

OR

Lawrence Livermore National Laboratory

Technical Information Department's Digital Library

http://www.llnl.gov/tid/Library.html 


\title{
Applications of sensitivity analysis to uncertainty quantification in variably saturated flow
}

\author{
C. Woodward ${ }^{\mathrm{a} *}, \mathrm{~K}$. Grant $^{\mathrm{b}}, \mathrm{R} \cdot$ Maxwell $^{\mathrm{c}}$
}

${ }^{a}$ Center for Applied Scientific Computing, Lawrence Livermore National Laboratory, P.O. Box 808, L-561, Livermore, CA 94588, USA

${ }^{\mathrm{b}}$ Atmospheric Sciences Division and Center for Applied Scientific Computing, LLNL

${ }^{\mathrm{c}}$ Geosciences and Environmental Technologies Division, LLNL

In this paper, we present results demonstrating the effectiveness of a sensitivity analysis approach to uncertainty quantification of a variably saturated flow model. The basis for our method is a software system which simultaneously solves for solutions of large-scale nonlinear systems of equations and the sensitivity of the solutions to selected parameters. We present test cases showing the effects on the relative uncertainty of pressure due to heterogeneity in the absolute permeability and to differences in parameterizing the Van Genuchten curve soil parameters, $\alpha$ and $n$.

\section{INTRODUCTION}

Simulation of water resource management problems often requires the solution of large problems with many spatial zones. In addition, effective use of simulation solutions requires knowledge of the uncertainty introduced into the solution by variances in problem data. Current techniques for obtaining this information can require many runs of the simulation code and can be very time-consuming, especially for large-scale problems.

Sensitivity analysis techniques give a way to compute solution uncertainties by using information on the sensitivities of the solution to various parameters. These sensitivities are just the solution derivative with respect to the parameter in question, and equations for them can be derived by differentiating the original model equation. The resulting sensitivity equation is linear and can be solved in tandem with the model equations. Solution uncertainties can be developed from these sensitivities with a straightforward additional calculation.

Our model for variably saturated flow is the mixed form of Richards' equation [1],

$\frac{\partial(s(p) \phi)}{\partial t}-\nabla \cdot\left(\frac{k k_{r}(p)}{\mu}(\nabla p-\rho g \nabla z)\right)=0$

where $s(p)$ is water saturation, $\rho$ is water density, $\phi$ is porosity of the medium, $k(x)$ is absolute permeability of the medium, $k_{r}(p)$ is relative permeability of water to air, $\mu$ is water viscosity, $g$ is gravity and $z$ is elevation.

*This work was performed under the auspices of the U.S. Department of Energy by University of California Lawrence Livermore National Laboratory under contract No. W-7405-Eng-48. 
Discretization is done for time with an implicit backward differencing scheme and for space with a cell-centered finite difference scheme. One-point upstream weighting is used for the face values of relative permeability and harmonic averaging for the absolute permeability. Applying these discretization schemes leads to a set of coupled discrete nonlinear equations that must be solved at each time step.

This paper presents the application of a software system for the computation of solutions to large, nonlinear systems of equations as well as the computation of the sensitivities of the solution to various input parameters to a variably saturated flow model. The solution sensitivities are then used to compute a first order estimate of the solution uncertainties based on uncertainties in the Van Genuchten parameters.

\section{UNCERTAINTY QUANTIFICATION and SENSITIVITIES}

In this section we describe how sensitivities can be used to estimate uncertainties in the context of variably saturated flow problems. Relative permeability and saturation as functions of $p$ can be modeled by Van Genuchten curves [2]. Often, the Van Genuchten curve soil parameters, $\alpha$ and $n$, are estimated using curve fits from data, thereby introducing error into the flow model. In addition, it is unclear as to how these parameters depend on the absolute permeability and whether this dependence impacts the problem solution. Thus, we model the $\alpha$ and $n$ parameters as,

$\alpha=a_{1} \ln |k|+a_{2} \quad$ and $\quad n=b_{1} \ln |k|+b_{2}$

where $k$ is the absolute permeability of the medium which can exhibit heterogeneity, and the $a_{i}$ and $b_{i}$ are uncertain parameters. The main questions we want to answer are: What is the uncertainty in the pressure caused by the uncertainties in the $a_{1}, a_{2}, b_{1}$, and $b_{2}$ parameters, and what is the sensitivity of pressure to changes in these parameters?

We assume that we have a random sample of size $N$ from the $\left(a_{1}, a_{2}, b_{1}, b_{2}\right)$ population. A direct Monte Carlo sampling approach would be to solve (1) $N$ times to find the mean and standard deviation of the resulting $N$ pressure fields. Instead, we first let

$\bar{a}_{j} \equiv \frac{1}{N} \sum_{i=1}^{N} a_{j, i} \quad$ and $\quad \bar{b}_{j} \equiv \frac{1}{N} \sum_{i=1}^{N} b_{j, i}$

be the corresponding sample means for $j=1,2$. We then solve (1) once using $a_{j}=\bar{a}_{j}$ and $b_{j}=\bar{b}_{j}$, denoting the nominal solution by $\tilde{p} \equiv p\left(\bar{a}_{1}, \bar{a}_{2}, \bar{b}_{1}, \bar{b}_{2}\right)$.

In the sensitivity analysis approach to uncertainty quantification, we use a first order Taylor series for $p\left(a_{1}, a_{2}, b_{1}, b_{2}\right)$ to approximate the dependence of $p$ on the parameters $a_{1}, a_{2}, b_{1}$ and $b_{2}$. That is, we use

$p\left(a_{1}, a_{2}, b_{1}, b_{2}\right) \approx \tilde{p}+\sum_{j=1}^{4}\left(\frac{\partial \tilde{p}}{\partial \gamma_{j}} \cdot\left(\gamma_{j}-\bar{\gamma}_{j}\right)\right)$,

where $\gamma_{j} \in\left[a_{1}, a_{2}, b_{1}, b_{2}\right]$. The derivatives $\partial p / \partial a_{j}$ and $\partial p / \partial b_{j}$ are called the sensitivities of $p$ with respect to $a_{j}$ and $b_{j}$. Equations for these derivatives can be obtained by differentiating (1) with respect to the $a_{j}$ and $b_{j}$ parameters. 
Next, we want to use the above Taylor series approximation to obtain an estimate for the variance $s_{p}^{2}$ of the pressure about $\tilde{p}$ at each point in space. We define the vectors

$\Delta \gamma_{j} \equiv\left(\begin{array}{c}\gamma_{1, j}-\bar{\gamma}_{j} \\ \vdots \\ \gamma_{N, j}-\bar{\gamma}_{j}\end{array}\right), \quad$ and $\quad \Delta p \equiv\left(\begin{array}{c}p_{1}-\tilde{p} \\ \vdots \\ p_{N}-\tilde{p}\end{array}\right)$

Again using (4), we have

$\Delta p \approx\left[\Delta a_{1}, \Delta a_{2}, \Delta b_{1}, \Delta b_{2}\right] \cdot\left(\begin{array}{c}\frac{\partial \tilde{p}}{\partial a_{1}} \\ \frac{\partial \tilde{p}}{\partial a_{2}} \\ \frac{\partial \tilde{p}}{\partial b_{1}} \\ \frac{\partial \tilde{p}}{\partial b_{2}}\end{array}\right)$.

Using these relationships, we can write

$s_{p}^{2} \approx \hat{s}_{p}^{2} \equiv c^{T} V c, \quad$ where $\quad c^{T} \equiv\left(\frac{\partial \tilde{p}}{\partial a_{1}}, \frac{\partial \tilde{p}}{\partial a_{2}}, \frac{\partial \tilde{p}}{\partial b_{1}}, \frac{\partial \tilde{p}}{\partial b_{2}}\right)$

and

$$
V \equiv \frac{1}{N-1} \cdot\left[\begin{array}{cccc}
\Delta a_{1}^{T} \Delta a_{1} & \Delta a_{1}^{T} \Delta a_{2} & \Delta a_{1}^{T} \Delta b_{1} & \Delta a_{1}^{T} \Delta b_{2} \\
\Delta a_{2}^{T} \Delta a_{1} & \Delta a_{2}^{T} \Delta a_{2} & \Delta a_{2}^{T} \Delta b_{1} & \Delta a_{2}^{T} \Delta b_{2} \\
\Delta b_{1}^{T} \Delta a_{1} & \Delta b_{1}^{T} \Delta a_{2} & \Delta b_{1}^{T} \Delta b_{1} & \Delta b_{1}^{T} \Delta b_{2} \\
\Delta b_{2}^{T} \Delta a_{1} & \Delta b_{2}^{T} \Delta a_{2} & \Delta b_{2}^{T} \Delta b_{1} & \Delta b_{2}^{T} \Delta b_{2}
\end{array}\right]
$$

The matrix $V$ is an approximation to the covariance matrix $C\left(a_{1}, a_{2}, b_{1}, b_{2}\right)$. The derivatives in the vector $c$ are evaluated using calculated sensitivities.

Of course, the error $e=s_{p}^{2}-\hat{s}_{p}^{2}$ depends upon how well the linear Taylor series approximations used above describe the true nonlinear behavior of the uncertainties. One could also extend this linear approach to a higher order method in the natural way. For example, a quadratic approach would require three additional solves for the extra sensitivities and would generally be more accurate, but it would most likely still be much less expensive than a full Monte Carlo sampling approach.

\section{IMPLEMENTATION}

We have implemented a three-dimensional variably saturated flow model based on Richards' equation in the ParFlow software package [3]. The Richards' equation model uses the KINSOL inexact Newton-Krylov [4] software package to solve the nonlinear systems at each time step [5]. Each nonlinear Newton iteration is solved with GMRES [6] preconditioned with Schaffer's semi-coarsening multigrid [7] method implemented in the hypre preconditioning library [8]. Previous work has shown that this solution method is very effective for variably saturated flow problems [9].

After discretization, the nonlinear equation for each finite difference point $x_{i, j, k}$ at each time step can be written in the form

$F_{i, j, k}(p)=0$, 
where $F$ is the nonlinear function expressing the discrete form of (1), and $p$ is the vector of pressures at the new time level at the finite difference points. Thus, at each time step, we have the coupled nonlinear system

$F\left(p, a_{1}, a_{2}, b_{1}, b_{2}\right)=0$

to solve for all the discrete pressure values. Note that the dependence on the uncertain parameters has explicitly been included in this system even though these parameters enter the model through the expressions for relative permeability and saturation.

Next, we define $S_{j}=\tilde{\gamma}_{j} \frac{\partial p}{\partial \gamma_{j}}$ for $\gamma_{j} \in\left[a_{1}, a_{2}, b_{1}, b_{2}\right]$ as the scaled sensitivity of pressure to the parameter $\gamma_{j}$. The $\tilde{\gamma}_{j}$ are nominal values used only for scaling. Differentiating (7) with respect to each of the parameters gives the equation,

$\frac{\partial F}{\partial p} S_{j}+\tilde{\gamma}_{j} \frac{\partial F}{\partial \gamma_{j}}=0$

This differentiation gives a linear equation for each of the 4 sensitivities we seek.

We calculate the solutions to these equations with the sensitivity version of KINSOL [10]. This software package solves the nonlinear system at a time step, then uses the solution to form (8) for each of the four parameters. First, $\frac{\partial F}{\partial \gamma_{j}}$ and the Jacobian of $F$ given by $\frac{\partial F}{\partial p}$ are evaluated. SensKINSOL evaluates the derivatives of the $F$ with respect to the parameters, $\gamma_{j}$, by taking finite differences of $F$ as in

$\frac{\partial F}{\partial a_{1}} \approx \frac{F\left(p, a_{1}+\delta_{a_{1}}, a_{2}, b_{1}, b_{2}\right)-F\left(p, a_{1}-\delta_{a_{1}}, a_{2}, b_{1}, b_{2}\right)}{2 \delta_{a_{1}}}$,

and similarly for the other derivatives of $F$. One could also use automatic differentiation techniques, and future releases of SensKINSOL will provide basic interfaces to the automatic differentiation software, ADIC [11]. SensKINSOL then solves these systems using the same linear solver and preconditioner as is used in the solution of the nonlinear iterations.

\section{NUMERICAL RESULTS}

To explore the sensitivity and uncertainty of pressures, we have constructed a test case with a large, deep vadose zone and a long-term infiltration study [12]. The alluvial site was modeled both as an anisotropic homogeneous system (case A) and as two isotropic heterogeneous systems (cases B and C). In case B Van Genuchten parameters are not correlated to saturated hydraulic conductivity, and in case $\mathrm{C}, \alpha$ in (2) is correlated to saturated hydraulic conductivity but $n$ is not. These cases are summarized in Table 1. The domain geometry was $150 \mathrm{~m} \times 150 \mathrm{~m} \times 250 \mathrm{~m}$ with a trench of $3 \mathrm{~m} \times 150 \mathrm{~m} \times 250 \mathrm{~m}$ infiltrating in the upper left of the domain with a rate of $5 \mathrm{~m}^{3} / \mathrm{d}$. A $25 \times 15 \times 50$ grid was used with cell spacings of $6 \mathrm{~m} \times 10 \mathrm{~m} \times 5 \mathrm{~m}$. For the homogeneous cases, the saturated hydraulic conductivity was set to $3.6 \mathrm{~m} / \mathrm{d}$ in the $x$ and $y$ directions and $0.517 \mathrm{~m} / \mathrm{d}$ in the $z$ direction. For the two heterogeneous cases, the hydraulic conductivity was assumed to be isotropic and described by a correlated, Gaussian random field, generated numerically via the turning bands algorithm [13]. A geometric mean of $7.5 \mathrm{~m} / \mathrm{d}$ with correlation lengths in the $x, y$, and $z$ directions of $25 \mathrm{~m}, 12 \mathrm{~m}$, and $6 \mathrm{~m}$, respectively, and a variance of the log of hydraulic conductivity of 1.5 was used. For the heterogeneous cases, three realizations of permeability with different random seeds were simulated for comparison. 
Table 1

This table gives the values of $a_{1}, a_{2}$, and $b_{2}$ used in the variations on our basic test case. The $\%$ standard deviation used for parameter $c$ is denoted by $\sigma_{c}$. Note that $b_{1}$ and its standard deviation were taken as 0 for all cases.

\begin{tabular}{cllllllcc}
\hline Case & $a_{1}$ & $\sigma_{a_{1}}$ & $a_{2}$ & $\sigma_{a_{2}}$ & $b_{2}$ & $\sigma_{b_{2}}$ & $\mathrm{k}$ & Random Seed \\
\hline $\mathrm{A}$ & 0.0 & $0 \%$ & 9.0 & $10 \%$ & 1.5 & $1 \%$ & Hom. & N/A \\
$\mathrm{B}$ & 0.0 & $0 \%$ & 9.0 & $10 \%$ & 1.5 & $1 \%$ & Het. & 3 \\
$\mathrm{C}$ & 0.9927 & $10 \%$ & 6.9998 & $10 \%$ & 1.5 & $1 \%$ & Het. & 3 \\
\hline
\end{tabular}

Saturation fields are shown in Figure 1 at 260 days for the three cases. We see the effects of the trench infiltrating down into the vadose zone. Case $\mathrm{C}$ shows much more dependence on the heterogeneity in the permeability field than the other two. This increased dependence is due primarily to the dependence of $\alpha$ in (2) on $k$.
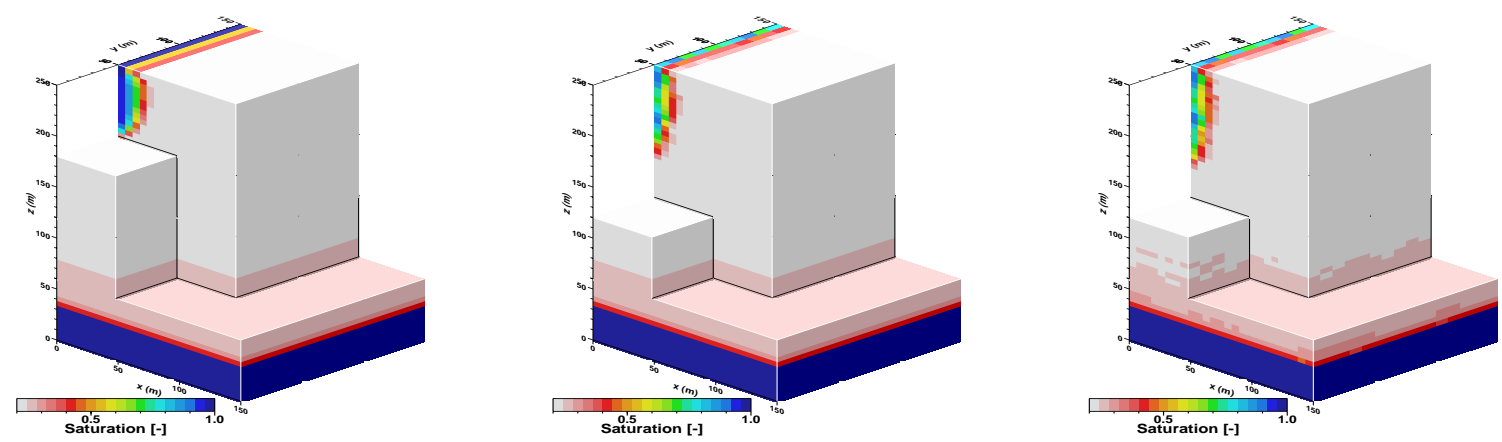

Figure 1. Saturation fields for the three cases at 260 days show varying impacts of heterogeneity. Case A (left) shows little impact, while cases B and C show increased impacts.

For cases $\mathrm{A}$ and $\mathrm{B}$, sensitivities were computed for $a_{2}$ and $b_{2}$, and for case $\mathrm{C}$, sensitivities were also computed for $a_{1}$. The sensitivities (unscaled) of pressure to $a_{1}, a_{2}$, and $b_{2}$ for case $\mathrm{C}$ are shown in Figure 2. We see that the sensitivity to $a_{1}$ is greater than to $a_{2}$ indicating that the heterogeneity is an important factor in the computed values of pressure. Also, the pressures are much more sensitive to $b_{2}$ than the other parameters. Cases A and B show the same relative sensitivity of the $a_{2}$ and $b_{2}$ parameters. This trend indicates that an accurate value of $n$ is much more critical to this test case than an accurate value of $\alpha$. We see this to be true in Table 2 where $\%$ uncertainties are shown for the three test cases at varying times. The final columns of this table give an estimate of the percent of the uncertainty contributed by a percent standard deviation given in Table 1 for each of the three parameters. Clearly $b_{2}$ with a $1 \%$ standard deviation contributes most to the uncertainty in the domain averaged pressure.

Table 2 also shows the $\%$ uncertainty of the domain averaged pressure for cases $\mathrm{B}$ and $\mathrm{C}$ with 



Figure 2. This figure shows unscaled sensitivities of pressure to changes in the $a_{1}, a_{2}$, and $b_{2}$ parameters. The figure indicates that $n$ is a much more important parameter to this test case than $\alpha$ in the Van Genuchten curves as noted by the different scales of absolute sensitivity for the three parameters in this case.

different seeds to the geostatistical model. Although we show results for only three realizations of the permeability field, we see that each of these realizations produces a similar mean and standard deviation of uncertainty when averaged across the domain, though local minima and maxima of uncertainty are realization dependent. There is indication of a slight but consistent decrease over time in the variance due to $b_{2}$.

Figure 3 shows the total relative uncertainties for the three cases at 260 days. These uncertainties were computed using the standard deviations given in Table 1 and (4). We see that uncertainties are much lower in the more saturated areas in the water table and near the trench. In these areas, of course, the relative permeabilities and saturations are less dependent on the parameters in the Van Genuchten curves. In addition, we see less uncertainties overall in case C indicating that accounting for the heterogeneity in the Van Genuchten parameters may reduce overall uncertainty in the final pressure solutions.

\section{CONCLUSIONS}

Solutions and solution sensitivities for variably saturated flow problems can be solved for simultaneously. In the case of Van Genuchten curves for relative permeability and saturation, sensitivity analysis has shown that our test case solutions are much more sensitive to $n$ than $\alpha$ and that incorporating heterogeneity in the formulation of $\alpha$ does not dramatically change estimates of uncertainty. In the future, the first order estimates of uncertainty computed here will be compared with a Monte Carlo simulation approach.

\section{ACKNOWLEDGMENTS}

The authors would like to thank Steven G. Smith for his valuable assistance with the ParFlow code package, Peter Brown for discussions on the computation and use of sensitivities, Steve 
Table 2

Spatially averaged percent uncertainties of pressure for different heterogeneity cases, random seeds, and times. The standard deviation of the percent uncertainty is denoted by $\sigma$. The final three columns show the approximate percentage contributions to the variance from parameters $a_{1}, a_{2}$, and $b_{2}$.

\begin{tabular}{lrrccccc}
\hline Case & Seed & Day & $\%$ Unc. & $\sigma$ & $\% \sigma_{a_{1}}^{2}$ & $\%_{0}^{2}{ }_{a_{2}}$ & $\% \sigma^{2} b_{2}$ \\
\hline A & N/A & 20 & $2.1956 \mathrm{e}+01$ & $2.8896 \mathrm{e}+00$ & 0.0 & 20.7 & 79.3 \\
$\mathrm{~A}$ & N/A & 60 & $2.1904 \mathrm{e}+01$ & $2.8837 \mathrm{e}+00$ & 0.0 & 21.0 & 79.0 \\
$\mathrm{~A}$ & N/A & 260 & $2.1472 \mathrm{e}+01$ & $3.4097 \mathrm{e}+00$ & 0.0 & 22.0 & 78.0 \\
$\mathrm{~B}$ & 3 & 20 & $2.1946 \mathrm{e}+01$ & $2.9223 \mathrm{e}+00$ & 0.0 & 20.7 & 79.3 \\
$\mathrm{~B}$ & 3 & 60 & $2.1837 \mathrm{e}+01$ & $3.0185 \mathrm{e}+00$ & 0.0 & 21.0 & 79.0 \\
$\mathrm{~B}$ & 3 & 260 & $2.1291 \mathrm{e}+01$ & $3.6151 \mathrm{e}+00$ & 0.0 & 22.3 & 77.7 \\
$\mathrm{~B}$ & 33 & 20 & $2.1962 \mathrm{e}+01$ & $2.8559 \mathrm{e}+00$ & 0.0 & 20.7 & 79.3 \\
$\mathrm{~B}$ & 33 & 60 & $2.1850 \mathrm{e}+01$ & $2.9706 \mathrm{e}+00$ & 0.0 & 21.0 & 79.0 \\
$\mathrm{~B}$ & 333 & 20 & $2.1991 \mathrm{e}+01$ & $2.7706 \mathrm{e}+00$ & 0.0 & 20.7 & 79.3 \\
$\mathrm{~B}$ & 333 & 60 & $2.1857 \mathrm{e}+01$ & $3.0048 \mathrm{e}+00$ & 0.0 & 20.9 & 79.1 \\
$\mathrm{C}$ & 3 & 20 & $2.1225 \mathrm{e}+01$ & $2.9623 \mathrm{e}+00$ & 1.0 & 13.9 & 85.0 \\
$\mathrm{C}$ & 3 & 60 & $2.1090 \mathrm{e}+01$ & $3.1476 \mathrm{e}+00$ & 1.1 & 14.1 & 84.8 \\
$\mathrm{C}$ & 3 & 260 & $2.0567 \mathrm{e}+01$ & $3.7458 \mathrm{e}+00$ & 1.1 & 15.0 & 83.9 \\
$\mathrm{C}$ & 33 & 20 & $2.1238 \mathrm{e}+01$ & $2.9062 \mathrm{e}+00$ & 1.0 & 14.1 & 84.9 \\
$\mathrm{C}$ & 33 & 60 & $2.1125 \mathrm{e}+01$ & $3.0217 \mathrm{e}+00$ & 1.0 & 14.3 & 84.7 \\
C & 333 & 20 & $2.1256 \mathrm{e}+01$ & $2.9161 \mathrm{e}+00$ & 1.0 & 14.0 & 84.9 \\
C & 333 & 60 & $2.1168 \mathrm{e}+01$ & $2.9783 \mathrm{e}+00$ & 1.1 & 14.2 & 84.7 \\
\hline
\end{tabular}

Carle for the development and assistance with the "Chunk" visualization package, and Andy Tompson for information on the test problem.

\section{REFERENCES}

1. L. A. Richards. Capillary conduction of liquids through porous mediums. Physics, 1:318333, 1931.

2. M. Th. van Genuchten. A closed form equation for predicting the hydraulic conductivity of unsaturated soils. Soil Sci. Soc. Am. J., 44:892-898, 1980.

3. C. S. Woodward. A Newton-Krylov-multigrid solver for variably saturated flow problems. In Computational Methods in Water Resources XII, volume 2, pages 609-616, Southampton, 1998. Computational Mechanics Publications.

4. P. N. Brown and Y. Saad. Hybrid krylov methods for nonlinear systems of equations. SIAM J. Sci. Statist. Comput., 11:450-481, 1990.

5. A. G. Taylor and A. C. Hindmarsh. User documentation for KINSOL, a nonlinear solver for sequential and parallel computers. Technical Report UCRL-ID-131185, Lawrence Livermore National Laboratory, 1998. 

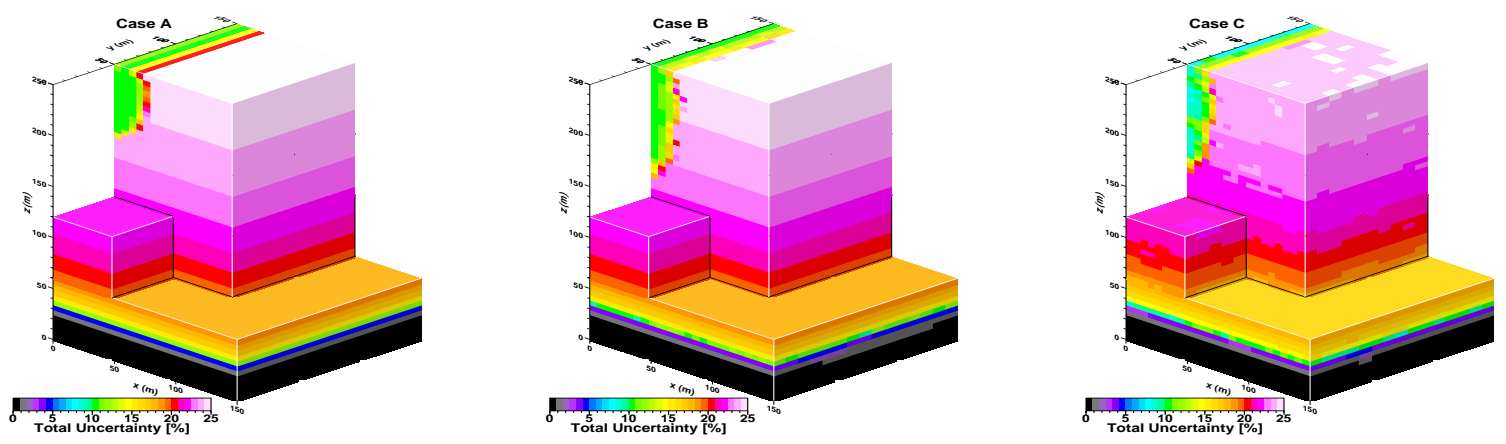

Figure 3. Percent uncertainties for the three cases show greater uncertainties away from saturated areas. Case $\mathrm{A}$ (left) has the highest uncertainty, and case $\mathrm{C}$ (right) has the least.

6. Y. Saad and M. H. Schultz. GMRES: A generalized minimal residual algorithm for solving nonsymmetric linear systems. SIAM J. Sci. Stat. Comput., 7(3):856-869, 1986.

7. S. Schaffer. A semi-coarsening multigrid method for elliptic partial differential equations with highly discontinuous and anisotropic coefficients. SIAM J. Sci. Comp., 20:228-242, 1999.

8. R. Falgout and U. Yang. hypre: a library of high performance preconditioners. In Proceedings for ICCS 2002, Lecture Notes in Computer Science. Springer Verlag, 2002.

9. J. E. Jones and C. S. Woodward. Newton-Krylov-multigrid solvers for large-scale, highly heterogeneous, variably saturated flow problems. Adv. Wat. Resour., 24:763-774, 2001.

10. K. E. Grant, A. C. Hindmarsh, and A. G. Taylor. User documentation for SensKINSOL, a variant of KINSOL for sensitivity analysis. Manuscript in preparation.

11. C. Bischof, L. Roh, and A. Mauer. ADIC - an extensible automatic differentiation tool for ANSI-C. Software-Practice and Experience, 27(12):1427-1456, 1997.

12. A. F. B. Tompson. Private communication. 2002.

13. A. Tompson, A. Ababou, and L. Gelhar. Implementation of the three-dimensional turning bands random field generator. Water Resour. Res., 25:2227-2243, 1989. 